\title{
Lactobacillus GG prevents recurrence of colitis in HLA-B27 transgenic rats after antibiotic treatment
}

\author{
L A Dieleman, M S Goerres, A Arends, D Sprengers, C Torrice, F Hoentjen, \\ W B Grenther, R B Sartor
}

\begin{abstract}
Background and aims: Bacteroides vulgatus induces colitis in gnotobiotic HLA- B27 transgenic (TG) rats while broad spectrum antibiotics prevent and treat colitis in specific pathogen free (SPF) TG rats although disease recurs after treatment ends. Lactobacilli treat human pouchitis and experimental colitis. We investigated if Lactobacillus rhamnosus GG (L GG) can prevent colitis in TG rats monoassociated with B vulgatus and if $L G G$ or Lactobacillus plantarum 299v (LP 299v) can treat established colitis in SPF TG rats and prevent recurrent disease after antibiotics were stopped.

Methods: Germfree B27 TG rats were monoassociated with B vulgatus for four weeks following two weeks of colonisation with L GG or no bacteria. SPF B27 TG rats received oral vancomycin and imipenem for two weeks, or water alone, followed by four weeks of treatment with oral LGG, LP 299v, or water only. Disease activity was quantified by blinded gross and histological scores, caecal myeloperoxidase (MPO) activity, and levels of interleukin (IL)-1 $\beta$, tumour necrosis factor (TNF), transforming growth factor $\beta$, and IL-10.

Results: $L G G$ did not prevent colitis in $B$ vulgatus co-associated TG rats or treat established disease in SPF rats. However, L GG but not LP 299v prevented colitis relapse in antibiotic treated rats with reduced gross and histological scores, caecal MPO, IL-1 $\beta$, and TNF whereas caecal IL-10 was increased.

Conclusions: L GG does not prevent colitis in gnotobiotic TG rats or treat established disease in SPF rats, but is superior to LP $299 \mathrm{v}$ in the prevention of recurrent colitis. These studies suggest that antibiotics and probiotic agents provide synergistic therapeutic effects, perhaps mediated by altered immunomodulation with selective activity of different lactobacillus species.
\end{abstract}

See end of article for authors' affiliations

Correspondence to: Dr L A Dieleman, Division of Digestive Diseases, Glaxo Bldg, Room 146, University of North Carolina at Chapel Hill, Chapel Hill, NC 27599-7038, USA Idiele@med.unc.edu

$\mathrm{T}$ he role of intestinal bacteria in the pathogenesis of human inflammatory bowel diseases (IBD), particularly Crohn's disease, is well recognised. ${ }^{12}$ These diseases typically occur at sites with the highest concentrations of intestinal bacteria, such as the colon and terminal ileum. Antibiotics and faecal diversion are effective treatment modalities for Crohn's disease ${ }^{1-3}$ whereas re-establishing continuity of the bypassed distal bowel or infusion of intestinal contents into the excluded ileum leads to disease recurrence. ${ }^{4}$

The role of intestinal bacteria in the initiation and perpetuation of chronic intestinal inflammation is most convincingly demonstrated in experimental models of chronic intestinal inflammation in which genetically susceptible hosts develop spontaneous colitis in the presence of commensal intestinal organisms whereas no disease occurs in the germ free (GF) state. $^{5-8}$

HLA-B27 transgenic (TG) rats develop colitis, gastritis, and systemic inflammation in a specific pathogen free (SPF) environment. ${ }^{9}$ However, GF TG rats and non-TG rats colonised with SPF bacteria show no evidence of colitis, gastritis, or arthritis. ${ }^{50}$ Colitis and gastroduodenal inflammation in TG rats is attenuated by metronidazole therapy and can be prevented and treated with broad spectrum antibiotics. ${ }^{11}$ Moreover, caecal bacterial overgrowth within an experimental blind loop exacerbates colitis whereas bypass of the caecum attenuates disease in this model, which correlates with concentrations of luminal bacteroides species. ${ }^{12}$ Bacteroides vulgatus preferentially induces colitis in TG rats after monoassociation for four weeks ${ }^{13}$ whereas monoassociation with Escherichia coli does not cause disease. These findings indicate that not all bacteria are equal in their capacity to induce colitis.
Probiotics are defined as living commensal microorganisms which are important to the health and well being of the host. ${ }^{14}$ Lactobacilli, although not predominant enteric organisms, are present throughout the gastrointestinal tract of healthy humans and rodents. Several lactobacillus strains, such as L plantarum 299v (LP 299v), L acidophilus, L fermentum, and Lactobacillus rhamnosus $G G$ ( $L G G$ ) can colonise the human gastrointestinal tract. ${ }^{15}$ Several human clinical trials have shown the efficacy of several species of probiotics in viral and bacterial induced infectious colitis as well as in antibiotic associated diarrhoea. ${ }^{14}{ }^{16} 17$ Rectal administration of native murine $L$ reuteri prevented colitis in interleukin (IL)-10 deficient mice and a similar effect was shown by the oral prebiotic lactulose, which stimulated the growth of endogenous lactobacilli species. ${ }^{18}$ Continuous oral treatment of $L P$ $299 v$ in GF IL-10 deficient mice colonised with SPF flora attenuated established colitis. ${ }^{19}$ A probiotic preparation including four species of lactobacilli, three bifidobacteria, and one Streptococcus salivarius (VSL\# 3) maintained remission of refractory pouchitis after transient antibiotic therapy. ${ }^{20}$ This probiotic cocktail VSL\#3 was also beneficial in the treatment of colitis in IL-10 deficient mice. ${ }^{21}$

The first aim of our study was to investigate if the probiotic species $L G G$ can prevent colitis in B27 TG rats monoassociated

Abbreviations: IBD, inflammatory bowel diseases; GF, germ free; TG, transgenic; SPF, specific pathogen free; CFU, colony forming units; $L G G$, Lactobacillus rhamnosus GG; LP 299v, Lactobacillus plantarum strain 299v; BBE, bacteroides bile esculin; ELISA, enzyme linked immunosorbent assay; IL, interleukin; PBS, phosphate buffered saline; TNF, tumour necrosis factor; TGF- $\beta$, transforming growth factor $\beta$. 
with the disease inducing $B$ vulgatus. Secondly, we determined if $L G G$ or $L P 299 v$ could treat established colitis in SPF B27 TG rats or prevent recurrent disease after broad spectrum antibiotics were stopped. Thirdly, we studied the protective mechanisms of probiotics in the prevention of relapse model.

\section{MATERIALS AND METHODS \\ Rats}

TG rats of the 33-3 line on an inbred F344 background, bearing several copies of the genes for HLA-B27 and human $\beta 2$ microglobulin, ${ }^{9}$ and their non-transgenic littermates were originally obtained from Dr Joel Taurog, Southwestern Medical School (Dallas, Texas, USA). After caesarean section they were housed in Trexler isolators in GF conditions at the Gnotobiotic Core facility at the College of Veterinary Medicine (Raleigh, North Carolina, USA). SPF TG rats and their non-TG littermates were born and maintained in filter top cages in a pathogen free environment.

\section{Bacteria}

Freeze dried cultures of $L G G$ and $L P 299 v$ were kindly provided by ConAgra (Omaha, Nebraska, USA).

Before administration, lactobacilli strains were grown aerobically overnight at $37^{\circ} \mathrm{C}$ in MRS broth. The next day bacteria were harvested by centrifugation. After washes, bacteria were resuspended in sterile phosphate buffered saline (PBS) and added to the drinking water. Quantification and purity of bacterial suspensions as bacterial colony forming units (CFU) was performed in serial dilutions of the drinking water on lactobacillus MRS-vancomycin plates. B vulgatus was kindly provided by $\mathrm{Dr} A B$ Onderdonk (Harvard University, Cambridge, Massachusetts, USA) and was originally derived from guinea pigs with carrageenan induced colitis. ${ }^{22}$ This strain has been used to induce colitis in monoassociated TG rats. ${ }^{13} \mathrm{~B}$ vulgatus was cultured anaerobically in BHI broth before colonisation of gnotobiotic rats.

\section{Experimental design}

Prevention study

In the first part of the study, GF TG rats and their non-TG littermates were divided into three groups of six rats each at the age of two months. The first two groups were monoassociated with either $B$ vulgatus or $L G G$ for four weeks, the third group was selectively colonised with $B$ vulgatus for four weeks but was colonised with $L G G$ for two weeks before co-colonisation with $B$ vulgatus. Colonisation with $B$ vulgatus was performed by oral and rectal swabbing using stool from other $B$ vulgatus monoassociated rats. $L G G$ or $L P 299 v$ was administered once in the drinking water at $10^{10} \mathrm{CFU}$ per rat per day after overnight culture at $37^{\circ} \mathrm{C}$ in MRS broth. The amount of bacteria consumed by each rat per day was calculated by measured consumption of $30 \mathrm{ml}$ of water per day times $3.3 \times 10^{8}$ $\mathrm{CFU} / \mathrm{ml}=10^{10} \mathrm{CFU}$. Successful colonisation was assessed after one week by plating stool suspensions on bacteroides bile esculin (BBE) and MRS plates for bacteroides spp and lactobacillus, respectively, for two days of anaerobic culture, as well as by performing Gram stains. No contaminating organisms were discovered. Rats were killed after four weeks of $B$ vulgatus or $L G G$ colonisation. At necropsy caeca and colons were fixed in $10 \%$ buffered formalin for histological evaluations. Caeca were also snap frozen for subsequent myeloperoxidase (MPO) and cytokine analysis. Caecal contents were collected for microbiological analysis, as described below.

\section{Prevention of relapse study}

At 10 weeks of age, SPF HLA-B27 TG rats were divided into the following four treatment groups, each consisting of six rats: group 1 received only drinking water ad libitum for six weeks; group 2 received $50 \mathrm{mg} / \mathrm{kg}$ each of vancomycin/imipenem in their drinking water for two weeks followed by four weeks of water; group 3 received $50 \mathrm{mg} / \mathrm{kg}$ each of vancomycin/ imipenem for two weeks followed by daily administration of $L$ $G G$ or $L P 299 \mathrm{v}$ in the drinking water at $10^{10} \mathrm{CFU} / \mathrm{rat} /$ day for four weeks; and group 4 received two weeks of water followed by four weeks of $10^{10} \mathrm{CFU} / \mathrm{rat} /$ day $L G G$ or $L P 299 \mathrm{v}$ in their drinking water. All rats were killed six weeks after the start of treatment. Caeca and colons were collected for histology and snap frozen for MPO and cytokine analysis, as described below. Caecal contents were collected for quantitative microbiological analysis.

\section{Histology}

Tissues were prepared as previously described. ${ }^{10} \mathrm{~A}$ validated histological inflammatory score ranging from 0 to 4 was used for blinded evaluation of colonic and caecal inflammation. ${ }^{10}$

\section{Caecal cytokine analysis}

Frozen caecal tissues were thawed and lysed in PBS containing a cocktail of protease inhibitors (antipain, aprotinin, leupeptin, pepstatin A, all at $1 \mu \mathrm{g} / \mathrm{ml}$ and $1 \mathrm{mM}$ PMSF; Sigma, St Louis, Missouri, USA). ${ }^{23}$ Tissue was then homogenised, after which the homogenate was assayed for IL-1 $\beta$, tumour necrosis factor (TNF), IL-10, and transforming growth factor $\beta$ (TGF- $\beta$ ). Concentrations of IL- $1 \beta$ were measured by a rat IL-1 $\beta$ enzyme linked immunosorbent assay (ELISA) developed by S Poole, as described previously. ${ }^{10}$ Caecal TNF and IL-10 concentrations were determined by rat cytokine specific ELISAs. TGF- $\beta 1$ concentrations were measured after acidification and neutralisation according to the manufacturer using a TGF- $\beta 1$ specific ELISA (Promega, Madison, Wisconsin, USA)

\section{MPO assay}

Homogenised caeca were assayed for MPO activity (units per gram of tissue), as described previously. ${ }^{24}$

\section{Determination of caecal bacterial concentrations}

Caecal contents were removed aseptically, immediately placed into an anaerobic chamber, and dissolved in sterile prereduced PBS. Serial 100-fold dilutions were plated and cultured anaerobically for two days on lactobacilli spp MRS vancomycin agar for quantification of lactobacillus, on BBE agar plates for bacteroides spp, and on Brucella agar (Remel, Lenexa, KS) for total anaerobic bacteria. Morphology was determined by Gram stain. Results are expressed as CFU per gram caecal contents dry weight.

\section{Inhibition of in vitro adherence to IEC-6 cells}

The rat epithelial cell line IEC-6 cells (ATCC \# CRL 1592; Rockville, Maryland, USA) were grown to confluence in a 12 well tissue culture plate in antibiotic free Dulbecco's modified Eagle's medium containing 5\% heat inactivated newborn bovine serum and $10 \mathrm{IU}$ (international units)/ml insulin (Life Technologies, Burlington, Ontario, Canada).

Intestinal epithelial adherence assay was performed according to Mack and colleagues. ${ }^{25}$ Briefly, after removal of non-adherent cells, an adherent IEC-6 monolayer was incubated at $37^{\circ} \mathrm{C}$ with $10^{\circ} \mathrm{CFU} B$ vulgatus in the presence or absence of $10^{9}, 10^{8}$, or $10^{7}$ CFU $L G G$ per well in $2 \mathrm{ml}$ of antibiotic free complete medium under aerobic conditions. After three hours of incubation, non-adherent bacteria were removed by washing three times with warm PBS, IEC-6 cells plus adherent bacteria were collected by trypsinisation. Collected cells and adherent bacteria were then serially diluted and plated anaerobically on BHI plates for 48 hours. Bacteria were counted by colony identification, confirmed by Gram staining.

\section{Statistical analysis}

Statistics were performed using the Student's $t$ test or MannWhitney test for comparison between groups. Significance was considered to be $\mathrm{p}<0.05$. All data are presented as mean (SEM). 


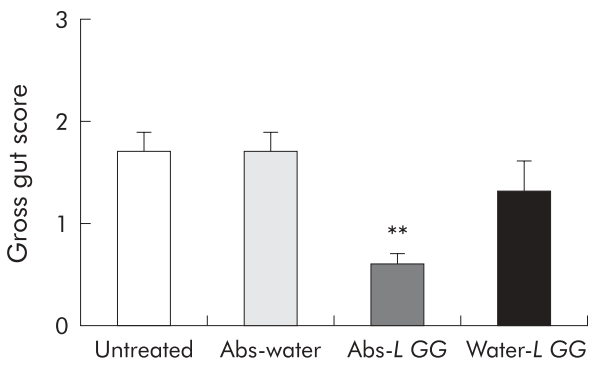

Figure 1 Gross gut scores (0-4) of caeca from specific pathogen free (SPF) transgenic (TG) rats treated with vancomycin/imipenem (Abs) or water followed by oral Lactobacillus rhamnosus GG (L GG) or water administration. Gross gut scores from untreated SPF TG rats are also given. Each group consisted of six rats. Data are expressed as mean (SEM). ${ }^{* *} p<0.01$ versus untreated control rats.

\section{RESULTS}

\section{Prevention study}

Monoassociation of TG rats with $B$ vulgatus resulted in mild caecal and colonic inflammation (histology score for the caecum $1.5(0.1)$ and colon $1.2(0.1))$. However, pretreatment of GF TG with $L G G$ followed after two weeks by co-association with $B$ vulgatus did not prevent the development of mild colitis induced by $B$ vulgatus (histology scores for the caecum 1.6 (0.2) and colon 1.1 (0.2)). However, monoassociation of gnotobiotic TG rats with $L G G$ did not induce colitis (caecal and colonic scores both $0.2(0.1))$. Similarly, gnotobiotic non-TG rats colonised with $B$ vulgatus, $L G G$, or both did not develop colitis (histology score for the caecum or colon 0.2 $(0.1))$.

Caecal IL-1 $\beta$ concentrations from $B$ vulgatus monoassociated TG rats (12 (3) $\mathrm{pg} / \mathrm{mg}$ caecal wet weight) were not significantly different from those obtained from $B$ vulgatus and $L$ GG co- associated TG rats ( 11 (2) pg/mg). Caecal IL-1 $\beta$ con-

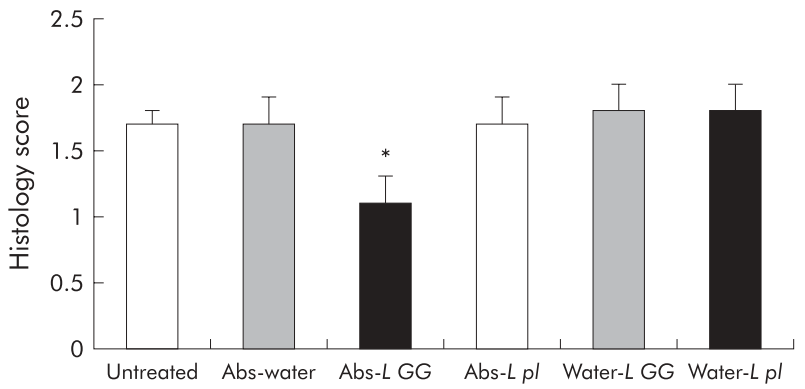

Figure 3 Blinded total colonic inflammatory scores in specific pathogen free (SPF) transgenic (TG) rats treated for two weeks with oral broad spectrum antibiotics (Abs) or water followed by four weeks of Lactobacillus rhamnosus GG (L GG), Lactobacillus plantarum strain 299v (L pl), or water. Also, histology scores from SPF TG untreated rats are given. Values represent mean (SEM) of total histology scores (0-4). Vancomycin/imipenem followed by daily oral $L G G$ administration significantly decreased total colonic histology scores versus other treatment groups. Feeding LP 299v did not prevent colitis relapse nor did it treat established colitis. ${ }^{*} p<0.05$ versus other treatment groups. Each group consisted of six SPF TG rats.

centrations from $L G G$ monoassociated TG rats (5 (2) ng/g) were similar to those from gnotobiotic non-TG littermates colonised with $B$ vulgatus, $L G G$, or both (2 (1) pg/mg).

The concentration of bacteroides spp in the caecal content of TG rats co-associated with $B$ vulgatus and $L G G$ did not differ significantly from $B$ vulgatus monoassociated TG rats (61 $(8) \times 10^{9}$ versus $47(4) \times 10^{9} \mathrm{CFU} / g$ caecal content in $B$ vulgatus and $B$ vulgatus plus $L G G$ associated TG rats, respectively). The concentration of caecal $L G G\left(0.45(0.04) \times 10^{9} \mathrm{CFU} / \mathrm{g}\right)$ in the $B$ vulgatus plus $L G G$ co-associated rats was 100 -fold lower than $B$ vulgatus concentrations but did not differ significantly from the concentration of caecal $L G G$ in $L G G$ monoassociated TG
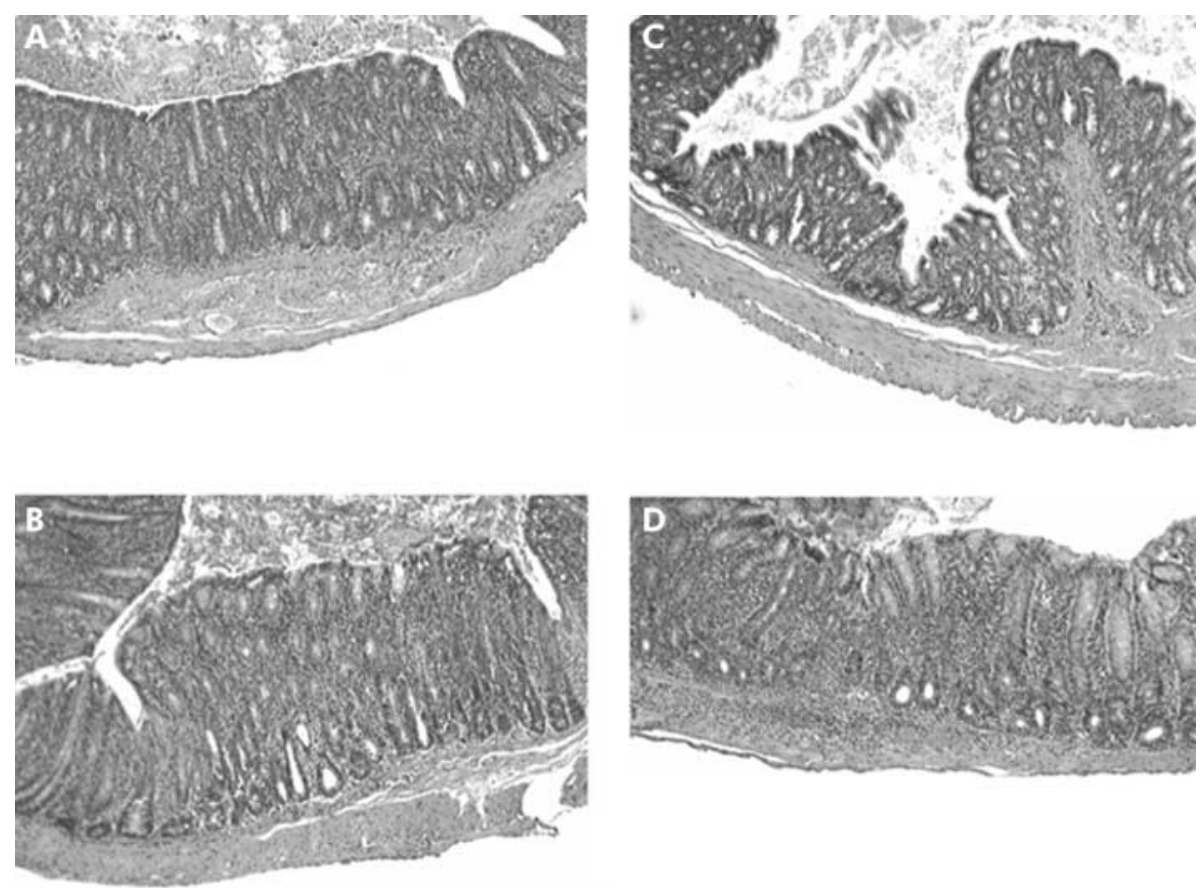

Figure 2 Representative photomicrographs of tissue sections $(\times 40)$ from caeca of 16 week old specific pathogen free (SPF) transgenic (TG) rats which were treated with: (A) water; (B) two weeks oral vancomycin/imipenem followed by four weeks of water; (C) two weeks oral of vancomycin/imipenem followed by daily administration of Lactobacillus rhamnosus GG (L GG) over four weeks; and (D) two weeks of water followed by four weeks of oral L GG. Note the extensive mucosal and some submucosal inflammation as well as significant crypt hyperplasia in caeca in (A), (B), and (D). Only mild to modest mucosal inflammation was seen in TG rats treated with the combination of broad spectrum antibiotics followed by $L G G(C)$. 


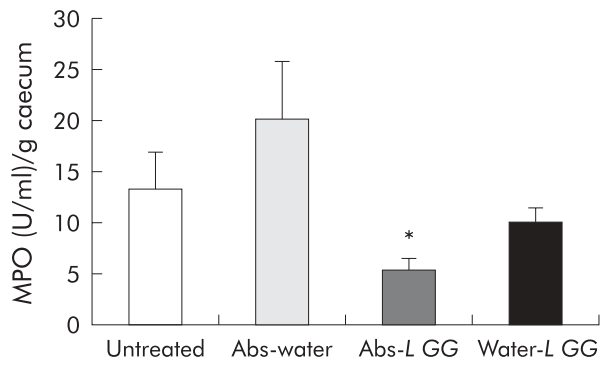

Figure 4 Caecal myeloperoxidase (MPO) concentrations in specific pathogen free (SPF) transgenic (TG) rats treated for two weeks with oral broad spectrum antibiotics (Abs) or water followed by four weeks of Lactobacillus rhamnosus GG (L GG), or water Values from untreated TG control rats are also given. Data represent mean (SEM) MPO levels in units per gram of caecum. ${ }^{*} p<0.05$ versus untreated controls.

rats $\left(0.49(0.10) \times 10^{9} \mathrm{CFU} / \mathrm{g}\right)$. Gram staining, and aerobic and anaerobic culture of caecal contents from gnotobiotic rats excluded contamination with other organisms.

\section{Prevention of colitis relapse in SPF B27 TG rats}

Oral administration of broad spectrum antibiotics (ora imipenem/vancomycin) for two weeks to SPF B27 TG rats followed by cessation of this treatment resulted in relapse of colitis after four weeks. Caecal gross gut scores $(1.7(0.2))$ as well as total colonic histology scores $(1.7(0.2))$ of antibioticwater treated rats four weeks after stopping antibiotics did not differ from untreated control TG animals (caecal gross score $1.7(0.2)$, total colonic histology score $1.7(0.1)$ ) (figs l-3). Also, $L G G$ administration alone could not effectively treat disease (caecal gross score $1.3(0.3)$, total colonic histology score $1.8(0.2))$. However, if antibiotic treatment was followed by daily oral administration of $L G G$, relapse of colitis could be partially prevented, as shown by a significant decrease in caecal gross score $(0.6(0.1))$ and total colonic histological inflammatory score $(1.1(0.3))$ of this treatment group (figs 1-3). In contrast with $L G G$ treatment, oral administration of LP 299v alone or after antibiotic treatment had no effect; neither caecal gross scores (data not shown) nor colonic histology scores (fig 3 ) differed significantly between treatment groups.

Caecal MPO values reflected the gross and histological scores; only TG rats treated with antibiotics followed by $L G G$ showed a significant reduction in caecal MPO (5.6 (1.0) U/g tissue) versus all other treatment groups whereas MPO in caecal homogenates from TG rats treated with antibiotics alone (20.3 (5.6) U/g) or with $L G G$ alone (10.2 (1.4) U/g) did not differ from untreated TG controls (13.3 (3.7) U/g) (fig 4). Caecal MPO from TG rats which had been fed $L$ plantarum with or without antibiotic pretreatment did not differ significantly from other treatment groups (data not shown).

\section{Mucosal cytokines}

Caecal homogenates from SPF TG rats treated with antibiotics and/or $L G G$ contained significantly less IL-1 $\beta$ than the other treatment groups (fig 5A). A similar pattern was shown for caecal TNF, although TNF concentrations were about 10-fold less than caecal IL-1 $\beta$ (fig 5B). Interestingly, IL-10 concentrations were significantly increased in caecal homogenates from antibiotics- $L G G$ treated TG rats versus other treatment groups whereas caecal TGF- $\beta$ levels were no different among the groups (fig 5B).

\section{Bacteriology of caecal contents}

No differences were found in total bacterial counts of caecal contents between the antibiotic and/or $L G G$ treatment groups in SPF TG rats. Also, concentrations of caecal anaerobic and aerobic bacteria, as determined by culture on Brucella and
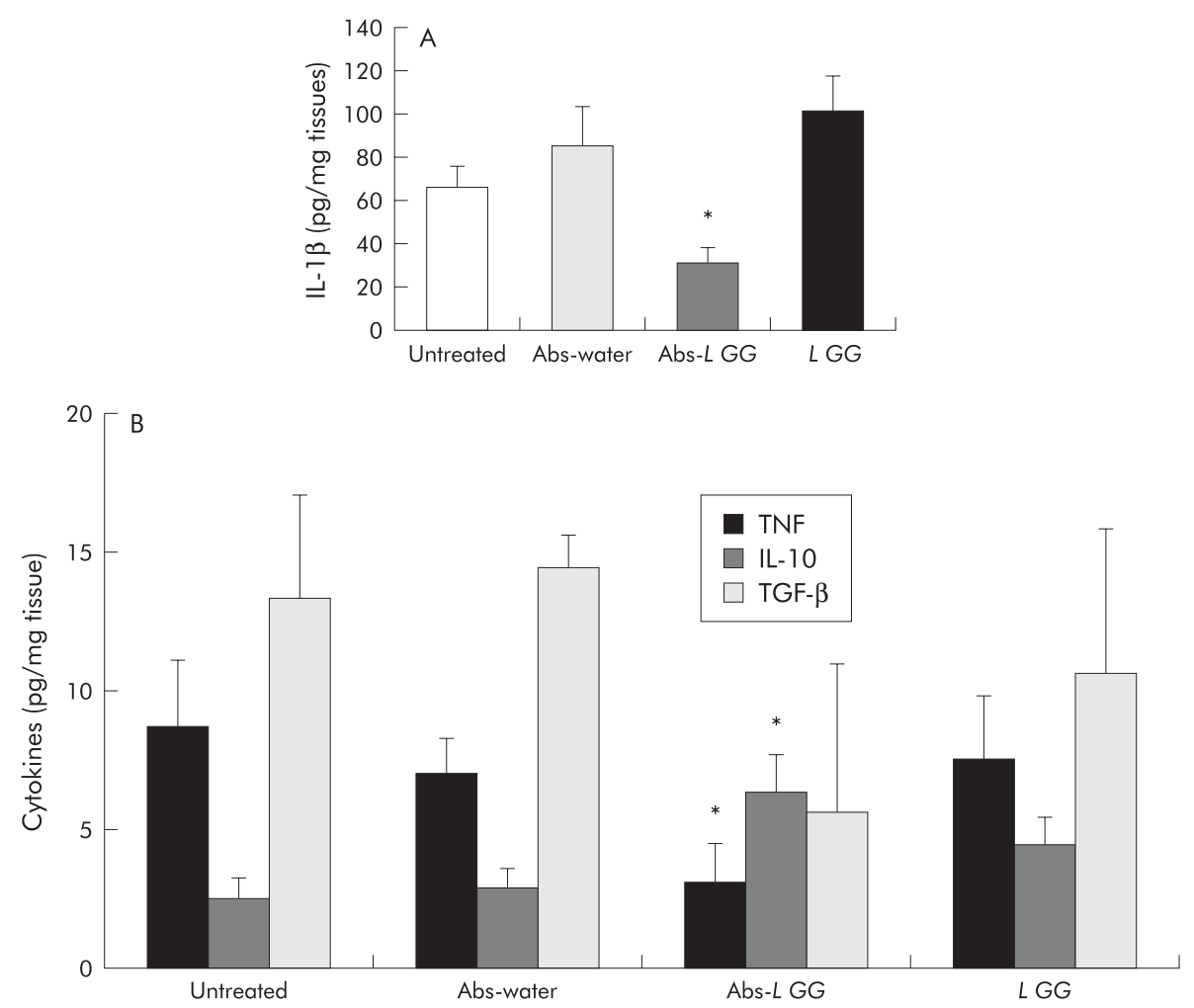

Figure 5 Caecal interleukin (IL)-1 $\beta$ (A), and tumour necrosis factor (TNF), IL-10, and transforming growth factor $\beta$ (TGF- $\beta$ ) (B) concentrations $(\mathrm{pg} / \mathrm{mg})$ from transgenic (TG) rats treated for two weeks with oral broad spectrum antibiotics (Abs) or water followed by four weeks of Lactobacillus rhamnosus GG (LGG) or water as well as from untreated controls. Values are mean (SEM). * $p<0.05$ versus untreated controls. The combination of antibiotics followed by L GG significantly decreased caecal IL-1 $\beta$ and TNF but also increased mucosal IL-10 concentrations. 


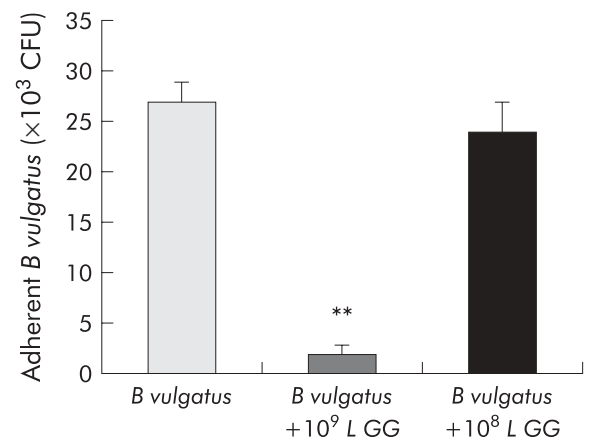

Figure 6 Dose dependent inhibition of in vitro adherence of $10^{\circ}$ CFU $B$ vulgatus $/ 2 \mathrm{ml}$ well to IEC- 6 cells by Lactobacillus rhamnosus GG (L GG). Data represent concentrations $\left(\times 10^{3} \mathrm{CFU}\right)$ of adherent $B$ vulgatus after three hours of incubation with IEC-6 monolayers in the presence or absence of $10^{8}$ or $10^{9} \mathrm{CFU} L G G / 2 \mathrm{ml}$ well. Values are mean (SEM). ${ }^{* *} p<0.01$ versus $B$ vulgatus control.

SBA plates, respectively, were not significantly different (data not shown). Oral administration of $L G G$ resulted in a 10 -fold increase in lactobacillus spp in caecal contents $\left(1.0(0.5) \times 10^{7}\right.$ CFU/g with antibiotics alone $v 12(3) \times 10^{7} \mathrm{CFU} / \mathrm{g}$ caecal content in antibiotics $-L G G$ treated rats). However, caecal bacteroides spp did not differ significantly among the treatment groups $\left(2.0(1.4) \times 10^{10}\right.$ with antibiotics alone $v 2.7(0.3) \times 10^{10} \mathrm{CFU} / \mathrm{g}$ caecal content in the antibiotics- $L G G$ treated group).

\section{In vitro inhibition of bacterial adherence}

$B$ vulgatus adhered to IEC-6 cells after an incubation of three hours. However, coculture of $10^{9} \mathrm{~B}$ vulgatus and $10^{9} \mathrm{~L} G G$ per well significantly reduced the amount of adherent $B$ vulgatus. No effect was noted with concentrations of $10^{8} /$ well (fig 6) or $10^{7} /$ well L GG (data not shown).

\section{DISCUSSION}

Broad spectrum antibiotics can prevent as well as treat established colitis in SPF B27 TG rats. ${ }^{11}$ Similar results were obtained in other experimental colitis models in rats and mice, ${ }^{11} 2627$ emphasising the important role of intestinal bacteria for induction and perpetuation of chronic intestinal inflammation. Oral vancomycin/imipenem treatment resulted in undetectable levels of bacteroides spp, which are capable of selectively inducing colitis in B27 TG rats. ${ }^{10} 13$

We found that colitis relapsed within four weeks of stopping vancomycin/imipenem treatment of established colitis in SPF TG rats, resulting in disease relapse within four weeks with gross and histological scores, MPO, and proinflammatory cytokine levels nearly identical to untreated controls. This finding correlated with the reappearance of luminal bacteroides spp as caecal bacteroides concentrations were not significantly different in antibiotic treated rats versus untreated TG controls. These findings are in agreement with clinical observations in Crohn's disease which recurs after stopping antibiotic treatment ${ }^{28}$ and can be explained by the reappearance of disease inducing intestinal bacteria in genetically susceptible hosts.

A clinically relevant finding of our study was that oral $L G G$ treatment partially prevented relapse of colitis after antibiotic treatment whereas $L G G$ administration alone failed to show a beneficial effect. This conclusion was supported by significantly decreased gross caecal inflammation and colonic histology scores, as well as a significant decrease in non-specific inflammatory markers and cytokines, such as caecal MPO, IL- 1 , and TNF in antibiotic- $L G G$ treated SPF TG rats versus other treatment groups. This is the first report of an interactive effect of antibiotic and probiotic therapy in experimental colitis. These results are in agreement with Gionchetti et al in which relapsing pouchitis was prevented in $85 \%$ of refractory pouchitis with an oral probiotic cocktail VSL\#3, which includes four strains of lactobacilli, three bifidobacteria species, and Streptococcus salivarius subsp thermophilus after the broad spectrum antibiotic rifaximin was stopped. ${ }^{20}$ Similarly, prospective treatment with rifaximin followed by VSL\#3 could also prevent endoscopic postoperative recurrence of Crohn's disease. ${ }^{29}$

Another major finding of our study was the specificity of prevention of relapsing colitis by various lactobacillus species, as demonstrated by the lack of efficacy of $L P 299 v$ in preventing colitis relapse in SPF TG rats after antibiotic treatment. However, this result is in contrast with our previous reports that oral LP 299v attenuated colitis in IL-10 KO mice, ${ }^{20}$ whereas $L G G$ had no effect. ${ }^{30}$ A preventive effect of $L P 299 v$ was also shown in methotrexate induced enterocolitis in rats. ${ }^{31}$ These contrasting responses suggest that different lactobacilli may have variable host specificity or different efficacy in various inflammatory conditions, indicating that not all probiotics are equally protective in chronic experimental colitis. The concept of selective dependency of beneficial effects of a probiotic bacterial species on the species and genetic background of the host is similar to that of disease inducing bacteria. ${ }^{10} 133233$

Other studies have reported primary beneficial effects of probiotics in the treatment or prevention of human or experimental intestinal inflammation. VSL\#3 was able to treat established colitis in IL-10 KO mice. ${ }^{25}$ The same cocktail has also shown efficacy in an open labelled study on maintenance therapy of ulcerative colitis. ${ }^{34} L G G$ can prevent relapsing Clostridium difficile diarrhoea, ${ }^{19}$ traveller's diarrhoea ${ }^{35}$ and rotavirus infections in children. ${ }^{36}$ The non-pathogenic $E$ coli strain Nissle was shown to be as effective as low dose mesalamine in maintaining remission of ulcerative colitis. ${ }^{37}{ }^{38}$

The protective mechanisms of probiotic bacteria are still relatively unknown, with postulated effects on luminal microecology, mucosal barrier function, and immunoregulation. In our study there was no decrease in the number of luminal bacteroides spp, raising the possibility that some of the protective effects of antibiotics- $L G G$ could be explained by immunomodulation. Not only does this therapy result in decreased caecal proinflammatory cytokines IL-1 $\beta$ and TNF, but it also increased mucosal IL-10. Lack of stimulation of mucosal IL-10 by $L G G$ or antibiotics alone indicate that neither treatment is responsible for induction of IL-10. It is possible that a combination of antibiotics and probiotics alters the balance of enteric bacterial components, leading to altered mucosal cytokine profiles. IL-10 is an immunoregulatory cytokine, mainly produced by macrophages, dendritic cells, and $\mathrm{T}$ regulatory $\mathrm{l}$ lymphocytes. However, we recently reported that in caecal bacterial lysate stimulated MLN cells of B27 TG rats as well as their non-TG littermates, IL-10 is primarily produced by B cells. ${ }^{39}$ In vitro, lysates of VSL\#3 stimulate IL-10 production and diminish TNF secretion by rat splenocytes stimulated with caecal bacteria. ${ }^{40}$ In preliminary data, it was demonstrated that DNA extracts of VSL\#3 induce IL-10 and exert protective immune responses. ${ }^{41}$ IL-10 can inhibit antigen specific proliferation and cytokine secretion by Thl lymphocytes and has downregulatory effects on macrophages and dendritic cells, such as suppression of activation and IL-12 production. ${ }^{42}{ }^{43}$ IL-10 can also prevent interferon $\gamma$ induced disruption of colonic epithelial barriers. ${ }^{44}$ Defective in vivo production of IL-10 results in spontaneous colitis in IL-10 KO mice in the presence of normal intestinal bacteria. ${ }^{75}$ Furthermore, oral administration of Lactococcus lactis, engineered to produce high colonic IL-10 levels, prevents the onset of colitis in IL-10 KO mice as well as in dextran sodium sulphate induced colitis. ${ }^{46}$ Thus stimulation of colonic IL-10 production by the combination of antibiotic use and $L G G$ colonisation could have a variety of beneficial effects on mucosal immunoregulation and barrier function.

Increased mucosal IL-10 production and decreased levels of the proinflammatory cytokines IL-1 $\beta$ and TNF by the 
combination of antibiotics and $L G G$ in our study are in agreement with the findings of Ulisse et al who showed increased IL-10 as well as decreased TNF and IFN- $\gamma$ in pouch biopsies of refractory pouchitis patients treated with VSL\#3 after antibiotic pretreatment. ${ }^{47}$ Similar to our findings, these authors found that tissue IL-10 levels were unchanged in inflamed pouches and were not affected by prior antibiotic treatment ${ }^{47}$ However, in IL-10 KO mice the probiotic cocktail VSL\#3 treated colitis with a concomitant decrease of mucosal IFN- $\gamma$ and TNF secretion, ${ }^{21}$ indicating that the beneficial effects of probiotics in colitis can be mediated by factors other than IL-10 induction.

Several non-immune protective mechanisms have been described for probiotic bacterial species. These organisms can exert growth inhibitory and bactericidal activities on enterotoxigenic $E$ coli.$^{48}$ However, in our study caecal concentrations of the disease inducing bacteroides spp were not significantly decreased. Another potential protective non-immune mechanism involves inhibition of epithelial adherence of disease inducing organisms. Intestinal bacteria compete with other organisms for a limited number of epithelial receptors. Increased amounts of colonic mucosal associated bacteria were found in IL-10 KO mice ${ }^{28}$ as well as in mucosal biopsies from IBD patients. ${ }^{49}$ Madsen et al also reported a relative lack of adherent lactobacilli in IL-10 $\mathrm{KO}$ mice $^{18}$ and restoration to normal levels after probiotic treatment. ${ }^{192}$ Our in vitro coculture studies demonstrated that $L G G$ decreased adherence of $B$ vulgatus to rat IEC-6 cells only at high concentrations of $10^{\circ}$ $\mathrm{CFU} / \mathrm{ml}$, with no effects at lower doses. This result is in agreement with in vitro studies by Madsen and colleagues ${ }^{21}$ and Mack and colleagues ${ }^{25}$ in which probiotic bacteria prevented the epithelial adherence of Salmonella dublin or enteropathogenic $E$ coli. However, in our study, in vivo co-association of $B$ vulgatus and $L G G$ did not prevent colitis in gnotobiotic TG rats. This lack of effect by $L G G$ in the dual association model could be explained by the overwhelming colonisation capacity of the disease inducing $B$ vulgatus versus $L G G$, which were 100 -fold more frequent than $L G G$. It is possible that at this relatively low in vivo luminal concentration $L G G$ would not be able to mediate protection by inhibiting epithelial adherence of $B \mathrm{vul}$ gatus, as equal concentrations of $L G G$ were required to interfere with $B$ vulgatus epithelial adherence. Based on these findings we postulate that protective effects by probiotics in SPF TG rats could only be achieved by pretreatment of rats with vancomycin/imipenem, which would result in a significant decrease in $B$ vulgatus ${ }^{11}$ before $L G G$ treatment. However, despite the prevention of colitis relapse by the combination treatment of vancomycin/imipenem followed by $L G G$, this treatment did not significantly reduce luminal bacteroides spp, even though increased caecal lactobacilli spp was shown by caecal culture. Taken together, reduction of luminal bacteroides spp could not explain the protective effects of broad spectrum antibiotics followed by $L G G$. Prevention of colitis relapse as well as immunomodulatory effects were shown only with the combination treatment of vancomycin/imipenem followed by $L G G$. It is possible that the combination treatment promoted synergistic interaction of $L G G$ with other endogenous probiotic species, resulting in disease protection. The absence of these potential beneficial interactions with other intestinal protective bacteria in gnotobiotic TG rats coassociated with $L G G$ and $B$ vulgatus could also explain the lack of efficacy of $L G G$ in the prevention of colitis. Alternatively, lack of exposure to lactic acid bacteria early in life in these gnotobiotic rats may prevent stimulation of protective responses by $L G G$ at an older age.

In summary, oral $L G G$ can prevent relapse of colitis in SPF B27 TG rats after antibiotics are stopped whereas oral administration of $L$ plantarum did not have any effect, demonstrating the selective protective effects of two lactobacillus species. The beneficial in vivo effects of $L G G$ following antibiotics are possibly the result of enhanced IL-10 production. This is the first study in experimental colitis in which antibiotics and probiotics display synergistic in vivo effects. Restoring the microbial balance between detrimental and protective luminal bacteria by combining antibiotic and probiotic approaches may be the most physiological approach to treat IBD and may alter the natural history of these chronic relapsing diseases.

\section{ACKNOWLEDGEMENTS}

This study was supported by NIH grants KO8 553773, RO3 558666, DK 40249, DK 34987, and by the Crohn's and Colitis Foundation of America. The authors wish to thank Charlotte Walters of the ImmunoTechnogy Core of the Center for Gastrointestinal Biology and Disease for technical assistance.

\section{Authors' affiliations}

L A Dieleman, M S Goerres, A Arends, D Sprengers, C Torrice, F Hoentien, W B Grenther, R B Sartor, Center for Gastrointestinal Biology and Disease, University of North Carolina at Chapel Hill, North Carolina, USA

\section{REFERENCES}

1 Fiocchi C. Inflammatory bowel disease: etiology and pathogenesis. Gastroenterology 1998:115:182-205.

2 Sartor RB. Microbial factors in the pathogenesis of Crohn's disease, ulcerative colitis and experimental intestinal inflammation, 5th edn. In: Kirsner J, ed. Inflammatory bowel diseases. Philadelphia: WB Saunders, 1999: 153-78.

3 Sutherland L, Singleton J, Sessions J. Double-blind, placebo-controlled trial of metronidazole in Crohn's disease. Gut 1991;32:1071-5.

4 D'Haens G, Geboes K, Peeters M, et al. Early lesions of recurrent Crohn's disease caused by infusion of intestinal contents in excluded ileum. Gastroenterology 1998;114:262-7.

5 Taurog JD, Richardson JA, Croft JT, et al. The germfree state prevents the development of gut and joint inflammatory disease in HLA-B27 transgenic rats. J Exp Med 1994; 180:2359-64.

6 Dianda L, Hanby AM, Wright NA et al. T cell receptor-alpha beta deficient deficient mice fail to develop colitis in the absence of a microbial environment. Am J Pathol 1997;150:91-7.

7 Sellon RK, Tonkonogy S, Schultz M, et al. Normal enteric bacteria are necessary for the development of spontaneous colitis and immune system activation in IL-10 deficient mice. Infect Immun 1998;66:5224-31.

8 Veltkamp C, Tonkonogy SL, de Jong YP, et al. Continuous stimulation by normal bacteria is essential for the development and perpetuation of colitis in Tge26 mice. Gastroenterology 2001;120:900-13.

9 Hammer RE, Maika SD, Richardson JA, et al. Spontaneous inflammatory disease in transgenic rats expressing HLA-B27 and human $\beta 2 \mathrm{~m}$ : An animal model of HLA-B27- associated human disorders. Cell 1990;63:1099-112.

10 Rath HC, Bender DE, Holt LC, et al. Normal bacteria stimulate colonic, gastric, and systemic inflammation in HLA-B27/b2m transgenic rats. J Clin Invest 1996;98:945-53.

11 Rath HC, Schultz M, Freitag R, et al. Different subsets of enteric bacteria induce and perpetuate experimental colitis in rats and mice. Infect Immun 2001;69:2277-85

12 Rath $\mathbf{H}$, lkeda J, Wilson $\mathrm{K}$, et al. Varying cecal bacterial loads influences colitis and gastritis in HLA-B27 transgenic rats. Gastroenterology 1999.116:310-19.

13 Rath H, Wilson K, Sartor R. Differential induction of colitis and gastritis in HLA-B27 transgenic rats selectively colonized with Bacteroides vulgatus or Escherichia coli. Infect Immun 1999:67:2969-74.

14 Gorbach SL. Probiotics and gastrointestinal health. Am J Gastroenterol 2000;95(suppl):S1-4

15 Bengmark S. Colonic food: pre- and probiotics. Am J Gastroenterol 2000;95(suppl): S5-7

16 Madsen KL. The use of probiotics in gastrointestinal disease. Can J Gastroenterol 2001:15:817-22.

17 Schultz M, Sartor RB. Probiotics and inflammatory bowel diseases. Am J Gastroenterol 2000;95(suppl):S19-21.

18 Madsen K, Doyle J, Jewell L, et al. Lactobacillus sp prevents developmen of enterocolitis in interleukin-10 gene deficient mice. Gastroenterology 1999;116:1107-14.

19 Schultz M, Veltkamp C, Dieleman L, et al. Lactobacillus plantarum 299v in the treatment and prevention of spontaneous colitis in interleukin-10 deficient mice Inflamm Bowel Dis 2002:8:71-80.

20 Gionchetti P, Rizzello F, Venturi A, et al. Oral bacteriotherapy as maintenance treatment in patients with chronic pouchitis: a double-blind placebo-controlled trial. Gastroenterology 2000;1 19:305-9.

21 Madsen K, Cornish A, Soper P, et al. Probiotic bacteria enhance murine and human intestinal barrier function. Gastroenterology 2001:121:580-91.

22 Onderdonk $\mathbf{A B}$, Franklin ML, Cisneros RL. Production of experimental ulcerative colitis in gnotobiotic guinea pigs with simplified microflora. Infect Immun 1981;32:225-31. 
23 Cominelli F, Nast C, Clark B, et al. Interleukin-1 (IL-1) gene expression, synthesis, and effect of specific IL- 1 receptor blockade in rabbit immune complex colitis. J Clin Invest 1990:86:972-80.

24 Grisham M, Benoit J, Granger D. Assessment of leukocyte involvement during ischemia and reperfusion of intestine. Methods Enzymol 1990; 186:729-42

25 Mack D, Michail S, Wei S, et al. Probiotics inhibit enteropathogenic E. coli adherence in vitro by inducing intestinal mucin gene expression. Am J Physiol 1999;276:G941-50.

26 Videla S, Vilaseca J, Guarner F, et al. Role of intestinal microflora in chronic inflammation and ulceration in the rat colon. Gut 1994:35:1090-7.

27 Madsen KL, Doyle JS, Tavernini MM, et al. Antibiotic therapy attenuates colitis in interleukin-10 gene-deficient mice. Gastroenterology 2000; 118:1094-105

28 Rutgeerts $\mathbf{P}$, Hiele M, Peeters $M$, et al. Controlled trial of metronidazole treatment for prevention of Crohn's disease recurrence after ileal resection. Gastroenterology 1995;108:1617-21.

29 Campieri M, Rizzello F, Venturi A, et al. Combination of antibiotic and probiotic treatment is efficacious in prophylaxis of post-operative recurrence of Crohn's disease: a randomized controlled study vs recurrence of Crohn's disease: a randomized contro

30 Veltkamp C, Tonkonogy S, Schultz $M$, et al. Lactobacillus plantarum is superior to Lactobacillus GG in preventing colitis in IL-10 deficient mice. Gastroenterology 1999;116:A838.

31 Mao Y, Nobaek S, Adawi D, et al. The effects of Lactobacillus strains and oat fiber on methotrexate-induced enterocolitis in rats. Gastroenterology 1996;111:334-44.

32 Dieleman L, Arends A, Tonkonogy S, et al. Helicobacter hepaticus does not induce or potentiate colitis in interleukin-10 deficient mice. Infect Immun 2000;68:5107-13

33 Kim S, Tonkonogy S, Balish E, et al. IL-10 deficient mice monoassociated with non- pathogenic Enteroccoccus faecalis develop chronic colitis. Gastroenterology 2001;120:A82

34 Venturi A, Gionchetti P, Rizzello F, et al. Impact on the composition of the faecal flora by a new probiotic preparation: preliminary data on maintenance treatment of patients with ulcerative colitis. Aliment maintenance treatment of patients with

35 Oksanen P, Salminen S, Saxelin M, et al. Prevention of travellers' diarrhoea by Lactobacillus GG. Ann Med 1990;22:53-6.

36 Isolauri E, Juntunen $M$, Rautanen $T$, et al. A human Lactobacillus strain (Lactobacillus casei sp strain GG) promotes recovery from acute diarrhea in children. Pediatrics 1991;88:90-7.
37 Kruis W, Schutz E, Fric $P$, et al. Double-blind comparison of an oral Escherichia coli preparation and mesalazine in maintaining remission of ulcerative colitis. Aliment Pharmacol Ther 1997;1 1:853-8.

38 Rembacken B, Snelling A, Hawkey P, et al. Non-pathogenic Escherichia coli versus mesalazine for the treatment of ulcerative colitis: a randomised trial. Lancet 1999;354:635-9.

39 Dieleman $L$, Tiwa $E$, Hoentien $F$, et al. B cells from mesenteric lymph nodes of resistant rats produce more interleukin- 10 than those from B27 transgenic rats following in vitro stimulation with cecal bacterial lysate. Gastroenterology 2002:122:A261.

40 Cender CJ, Haller D, Walters $C$, et al. VSL\#3 alters cytokine production of unfractionated splenocytes upon stimulation with cecal bacterial lysate: immunomodulation by this probiotic combination. Gastroenterology 2002;122:A145

41 Madsen $\mathrm{K}$, Jijon $\mathrm{H}$, Yeung $\mathrm{H}$, et al. DNA from probiotic bacteria exerts anti- inflammatory actions on intestinal epithelial cells by inhibition of NF-kb. Gastroenterology 2002;122:A64.

42 Moore KW, O'Garro A, de Waal Malefyt R, et al. Interleukin-10. Ann Rev Immunol 1993;11:165-90.

43 Tripp CS, Wolf SF, Unanue ER. Interleukin 12 and tumor necrosis factor alpha are costimulators of interferon gamma production by natural killer cells in severe combined immunodeficiency mice with listeriosis, and interleukin 10 is a physiologic antagonist. Proc Natl Acad Sci USA 1993;90:3725-9

44 Madsen K, Lewis S, Tavernini $M$, et al. Interleukin 10 prevents cytokine-induced disruption of T84 monolayer barrier integrity and limits chloride secretion. Gastroenterology 1997;113:151-9.

45 Kuhn R, Lohler J, Rennick D, et al. Interleukin-10 deficient mice develop chronic enterocolitis. Cell 1993;75:263-74.

46 Steidler L, Hans W, Schotte L. Treatment of murine colitis by Lactococcus lactis secreting interleukin-10. Science 2000;289:1352-5.

47 Ulisse S, Gionchetti P, D'Alo S, et al. Expression of cytokines, inducible nitric oxide synthetase, and matrix metalloproteinases in pouchitis. Am J Gastroenterol 2001;96:2691-9.

48 Gopal P, Prasad J, Smart J, et al. In vitro adherence properties of Lactobacillus rhamnosus DR20 and Bifidobacterium lactis DR 10 strains and their antagonistic activity against an enterotoxigenic Escherichia coli. Int J Food Microbiol. 2001;67:207-16.

49 Swidsinski A, Ladhoff A, Pernthaler A, et al. Mucosal flora in inflammatory bowel disease. Gastroenterology 2002;122:44-54 